\title{
Genome-wide association of trajectories of systolic blood pressure change
}

\author{
Anne E. Justice ${ }^{1 * \dagger}$, Annie Green Howard ${ }^{2 \dagger}$, Geetha Chittoor ${ }^{3 \dagger}$, Lindsay Fernandez-Rhodes ${ }^{1}$, Misa Graff ${ }^{1}$, \\ V. Saroja Voruganti ${ }^{3}$, Guoqing Diao ${ }^{4}$, Shelly-Ann M. Love ${ }^{1}$, Nora Franceschini', Jeffrey R. O'Connell', \\ Christy L. Avery ${ }^{1}$, Kristin L. Young ${ }^{1}$ and Kari E. North ${ }^{1}$
}

From Genetic Analysis Workshop 19

Vienna, Austria. 24-26 August 2014

\begin{abstract}
Background: There is great interindividual variation in systolic blood pressure (SBP) as a result of the influences of several factors, including sex, ancestry, smoking status, medication use, and, especially, age. The majority of genetic studies have examined SBP measured cross-sectionally; however, SBP changes over time, and not necessarily in a linear fashion. Therefore, this study conducted a genome-wide association (GWA) study of SBP change trajectories using data available through the Genetic Analysis Workshop 19 (GAW19) of 959 individuals from 20 extended Mexican American families from the San Antonio Family Studies with up to 4 measures of SBP. We performed structural equation modeling (SEM) while taking into account potential genetic effects to identify how, if at all, to include covariates in estimating the SBP change trajectories using a mixture model based latent class growth modeling (LCGM) approach for use in the GWA analyses.
\end{abstract}

Results: The semiparametric LCGM approach identified 5 trajectory classes that captured SBP changes across age. Each LCGM identified trajectory group was ranked based on the average number of cumulative years as hypertensive. Using a pairwise comparison of these classes the heritability estimates range from 12 to $94 \%$ (SE = 17 to $40 \%$ ).

Conclusion: These identified trajectories are significantly heritable, and we identified a total of 8 promising loci that influence one's trajectory in SBP change across age. Our results demonstrate the potential utility of capitalizing on extant genetic data and longitudinal SBP assessments available through GAW19 to explore novel analytical methods with promising results.

\section{Background}

There is great interindividual variation in systolic blood pressure (SBP) as a result of the influences of several factors, including sex, ancestry, smoking status, medication use, stress, socioeconomic status, and, especially, age [1]. Studies estimate strong genetic effects when examining SBP cross-sectionally $\left(h^{2}=0.42\right)$, with even higher estimates for longitudinal measures of SBP change $\left(h^{2}=0.57\right)$ [2]. Yet, there are few published largescale genetic studies that have leveraged longitudinal

\footnotetext{
* Correspondence: anne.justice@unc.edu

${ }^{\dagger}$ Equal contributors

'Department of Epidemiology, University of North Carolina, Chapel Hill, NC 27514, USA

Full list of author information is available at the end of the article
}

measures of blood pressure (BP) $[3,4]$. Newer studies that have partitioned heterogeneous phenotypes into meaningful subphenotypes have proven useful for the identification of novel genetic susceptibilities and have allowed for previously missing heritability to be detected in complex disease traits (eg, cancer, autism, schizophrenia) [5-8]. These studies suggest that methodological innovations that identify homogenous subphenotypes may be useful for characterizing longitudinal BP change, yet this avenue remains largely unexplored in the genetic literature.

One possibility for identifying subphenotypes of BP change is through a group-based trajectory analysis. Trajectories can take advantage of longitudinal data to create a new outcome variable to summarize a unique component of the change in the phenotype, thereby 
minimizing trait heterogeneity, and allowing for a new trait to be considered in quantitative genetic analyses, which may allow for the identification of clinically relevant genetic biomarkers for disease progression and prognosis, such as hypertension [9]. Structural equation modeling (SEM) can help to show if certain covariates relate to the change trajectories, which will decrease bias while increasing precision and accuracy in genome-wide association (GWA) study analyses. Therefore, this study aims to conduct a GWA study of SBP change trajectories using data available through the Genetic Analysis Workshop 19 (GAW19). We first used previously identified BP single nucleotide polymorphisms (SNPs) to perform SEM and model the assumed underlying relationships between variables while taking into account potential genetic effects. Then these SEM results were used to inform a semiparametric latent class growth modeling (LCGM), which was used to identify distinct groups of SBP change trajectories within the population, using a widely available statistical package (PROC TRAJ for SAS) $[10,11]$, for use in the GWA study analyses.

\section{Methods}

\section{Materials}

GAW19 data were provided by the Type 2 Diabetes Genetic Exploration by Next-generation sequencing in Ethnic Samples (T2D-GENES) Consortium Project 2. Participants' genetic and phenotypic data were drawn from 959 individuals from 20 Mexican American families as part of the San Antonio Family Studies (SAFS) [12].

\section{Phenotypes}

Our analysis focused on SBP, where SBP was corrected for BP-lowering medication by adding a constant to all SBP measures that reported medication use (SBP + $15 \mathrm{~mm} \mathrm{Hg}$ ) [13]. Only individuals with a minimum of 2 SBP measures who did not exhibit greater than 3 standard deviations of change in any SBP measure were used in the final trajectory and association analyses $(N=683)$. Each time-varying measure was collected at 4 time points across 17 years; however, all subjects with at least 2 measurements were included as these methods assume missing data are missing completely at random.

\section{Single nucleotide polymorphism selection}

Genome-wide data for 472,049 SNPs genotyped at Texas Biomedical Research Institute on the Illumina Infinium Bead chips: HumanHap550v3, supplemented with HumanExon510Sv1; Human660W-Quadv1; Human1Mv1; and Human1M-Duov3 arrays on odd-numbered autosomes were provided for analysis. All SNPs used in the analyses were filtered for Mendelian errors, monomorphic SNPs [12], and Hardy-Weinberg equilibrium. Merlin was used to impute missing genotypes. For the SEM model prediction, we extracted previously identified SBP, diastolic blood pressure (DBP), and pulse pressure-associated SNPs [14-19] from previous GWA studies data and considered each SNP separately. SNPs were only included in SEM if each genotype had at least 30 individuals. The full genetic panel, including imputed genotypes, was used in the trajectory association analyses.

\section{Structural equation modeling}

We used SEM to identify covariates to include in estimating the SBP change trajectories. SEM is used here to determine if potential covariates are associated with trajectory class membership or associated with deviations from the assigned class-specific trajectory to better determine how and if covariates should be used in the LCGM. For the SEM component (Mplus v7.11), we defined a structure a priori to evaluate at what time points certain covariates directly and indirectly impacted SBP in separate SNP models, using previously identified SNPs extracted from the full genome-wide data. A lag effect for SBP was included to allow for current values to be related to values of the time point immediately prior, thereby allowing for covariates to have an indirect effect on SBP through their impact on a previous time point. Figure 1 illustrates the full SEM model, including direct and indirect covariate effects tested. Generalized estimating equations were used within the SEM framework to account for the correlation within an individual and within a pedigree. Root mean square error of approximation (RMSEA) as well as comparative fit index (CFI), and Tucker-Lewis index (TLI) were examined to ensure appropriate model fit. Good model fit was defined as RMSEA $<0.06$, and CFI and TLI values close to 1.0 [20-22]. Only those variables that were significant in at least 2 different SNP models (Fig. 1) and at multiple time points for each of these models were included in the final LCGM analysis; however, other variables were considered for adjustment in the association analysis (eg, principal components [PCs] to control for ancestry).

\section{Latent class growth modeling}

We used a semiparametric LCGM approach to identify distinct groups of developmental trajectories [10, 23, 24]. Identification of SBP trajectories was done assuming a censored normal distribution using multivariate mixture model implemented in PROC TRAJ in SAS version 9.2. This model assumes that given class membership, the repeated measurements for the $i^{\text {th }}$ individual are independent [10]. SBP was modeled across age, including data from all 4 time points. Significant covariates in the SEM models were included to account for their impact on an individual's trajectory. Final models were selected using the Bayesian information criterion (BIC) as well as practical considerations such as group size $(N>25)$ [25], 


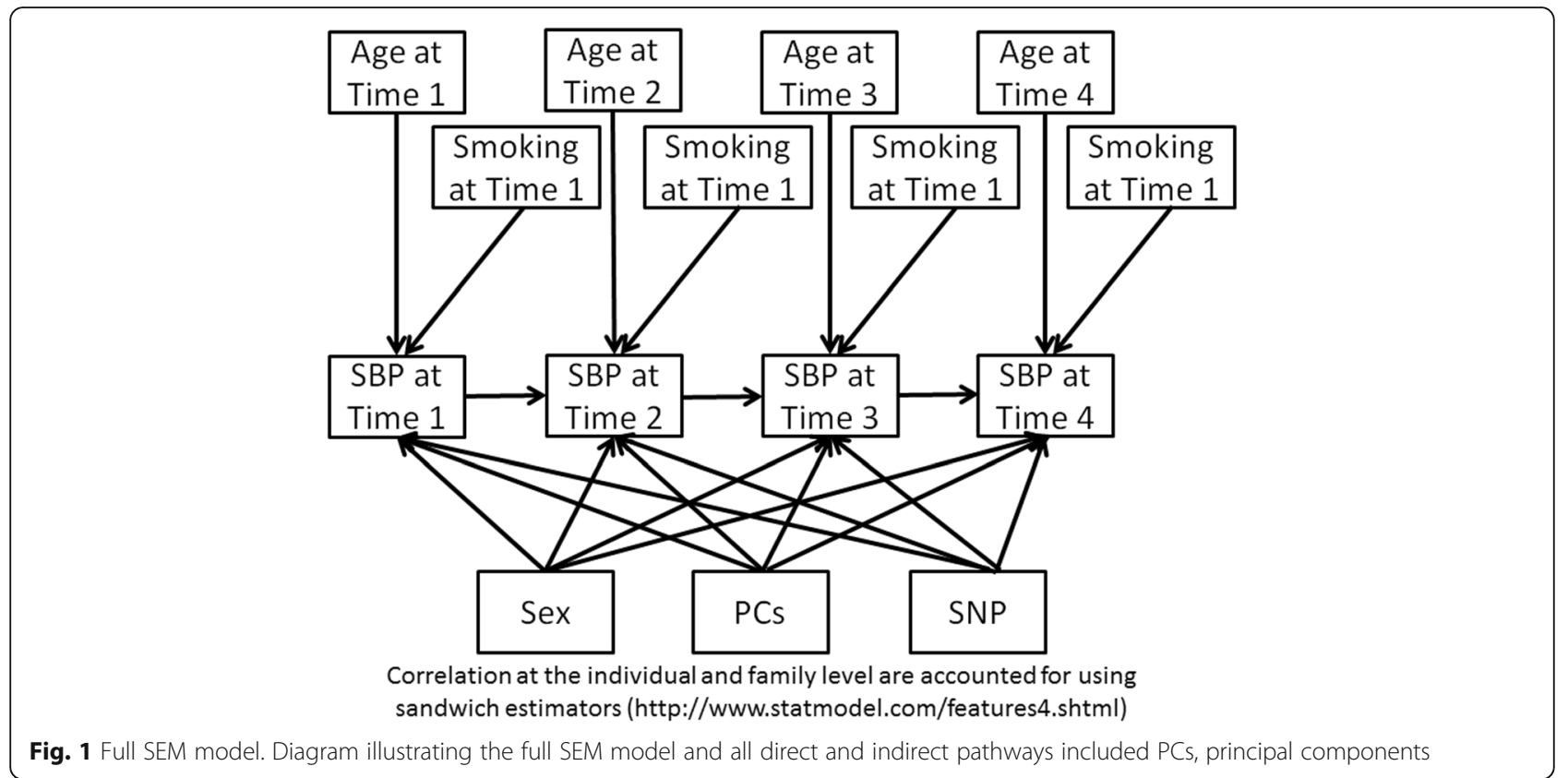

uniqueness, and interpretability of classes. During model selection all trajectories were assumed to follow the same order polynomial. However, once the best model was selected with regards to the number of classes and overall order of polynomial, we assessed the impact of the order of the polynomial for each class on model fit and retained only significant intercept, linear, and quadratic terms. The identified trajectory classes were assigned at the individual level, based on the class with the highest predicted posterior probability of class membership [25].

\section{Genome-wide association analyses}

Each LCGM identified trajectory group was ranked based on health risk, defined as the average number of cumulative years as hypertensive (ie, members of group 1 exhibiting the fewest number of hypertensive years and group 5 the greatest). We used PC scores to model differences in ancestral contributions among study participants. PCs were calculated using the unrelated founders and a subset of 28,156 independent $\left(r^{2}<0.2\right)$ SNPs [26]. The resulting top 4 PCs were included as additional covariates in association analyses, as previous analyses found these sufficient for controlling population substructure [27]. We used SOLAR (Sequential Oligogenic Linkage Analysis Routines) [28] to estimate heritability. For GWA analysis on the trajectory group membership variables, we used MMAP, an open source software package written in C for genetic association analysis in both population-based and family data using linear mixed models. MMAP uses variance components within the mixed model framework to

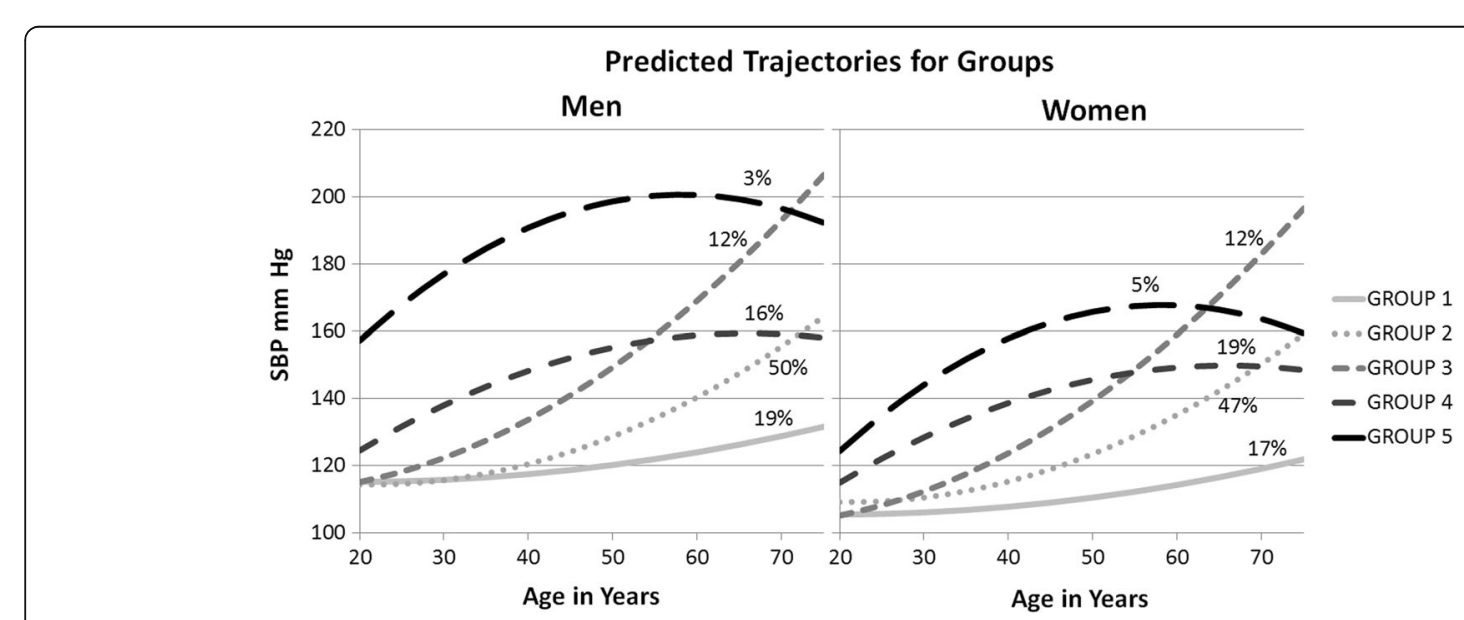

Fig. 2 Fitted trajectories. Fitted trajectories by sex for each group identified in the LCGM analysis 


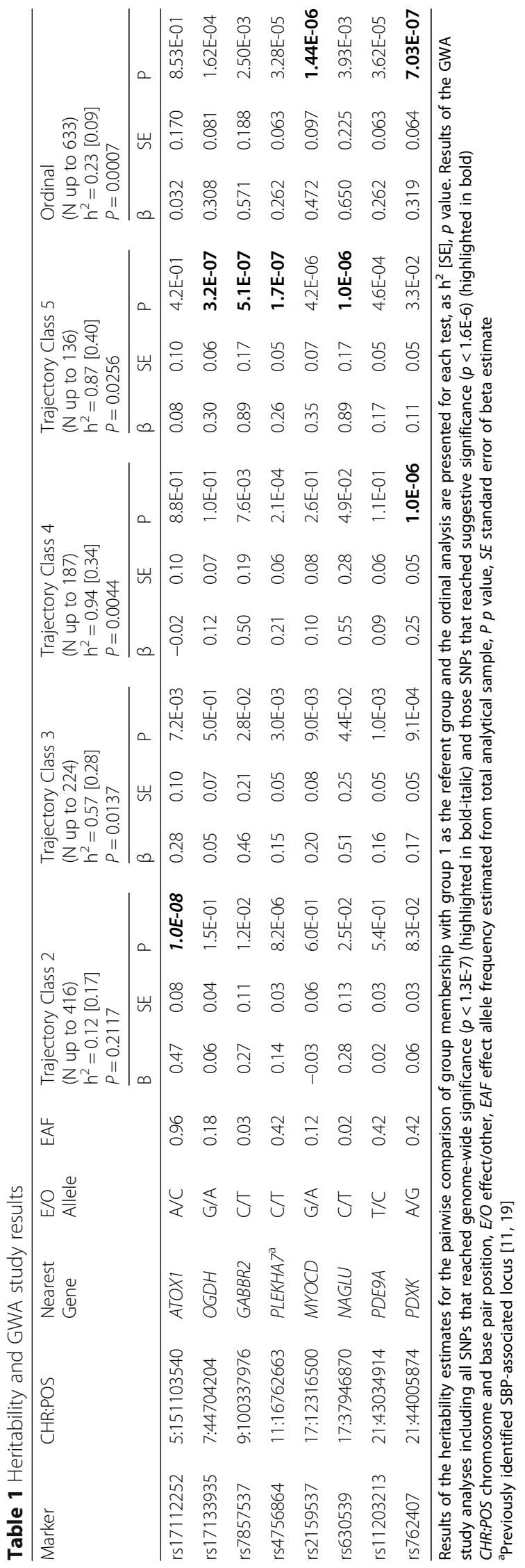


account for relatedness between individuals. For GWA analyses, we assumed an additive genetic model, and included the first 4 PCs as fixed effects to control for population structure [29]. We conducted both pairwise GWA analyses between groups with group 1 as the referent group. Also, to take advantage of the full data set, we conducted a GWA treating the rank ordered trajectories as a continuous trait in MMAP, coded as 1 through 5. Results were deemed genome-wide significant (GWS) when $p<$ $1.3 \mathrm{E}-7$ and suggestive when $p<1.6 \mathrm{E}-6$, based on previous San Antonio Family Studies [30] simulations.

\section{Results}

\section{Structural equation modeling}

The final SEM models included a total of 19 previously identified BP-associated SNPs available in the GAW19 data set to identify mediators and effect modifiers to include in the identification of trajectory classes. Four of the established BP SNPs displayed a nominally significant $(p<0.01)$ association with SBP at 1 or more time points, and were investigated further for potential effect mediators. The SEM led to the inclusion of sex in the LCGM and the consideration of PCs in the GWA (data not shown).

\section{Latent class growth modeling}

As the difference in SBP trajectories between men and women were not of primary interest for this study and sex impacted SBP directly and indirectly at multiple time points in the SEM, we adjusted for sex at each time point in the LCGM. Class prediction was performed requesting 1 to 6 groups, with the highest BIC resulting from assignment of 5 groups with age as a quadratic function in all classes (Fig. 2).

\section{Heritability and covariate selection}

Table 1 summarizes the results of the heritability estimates and displays the significant PCs for each pairwise group comparison. The heritability estimates range from 0.12 to 0.94 , standard errors (SE) from 0.17 to 0.40 (Table 1). PC2 and PC3 were both significant predictors of group comparisons in 1or more models.

\section{Genome-wide association}

A quantile-quantile $(\mathrm{Q}-\mathrm{Q})$ plot of the final GWA results for all 5 approaches exhibits no strong evidence for genomic inflation (Fig. 3). After filtering on minor allele frequency (MAF) of greater than $1 \%$, association analyses identified one GWS $(p<1.3 \mathrm{E}-7)$ SNP near the ATOX1 gene (rs17112252, $p=1 \mathrm{E}-8)$ for the pairwise association analysis of trajectory group 2 (see Table 1). Although no other GWA study analysis resulted in a GWS association, an additional 7 SNPs reached suggestive significance $(p<1.6 \mathrm{E}-6)$ in 1 or more GWA studies, including rs4756864 for the group near the PLEKHA7 gene, only

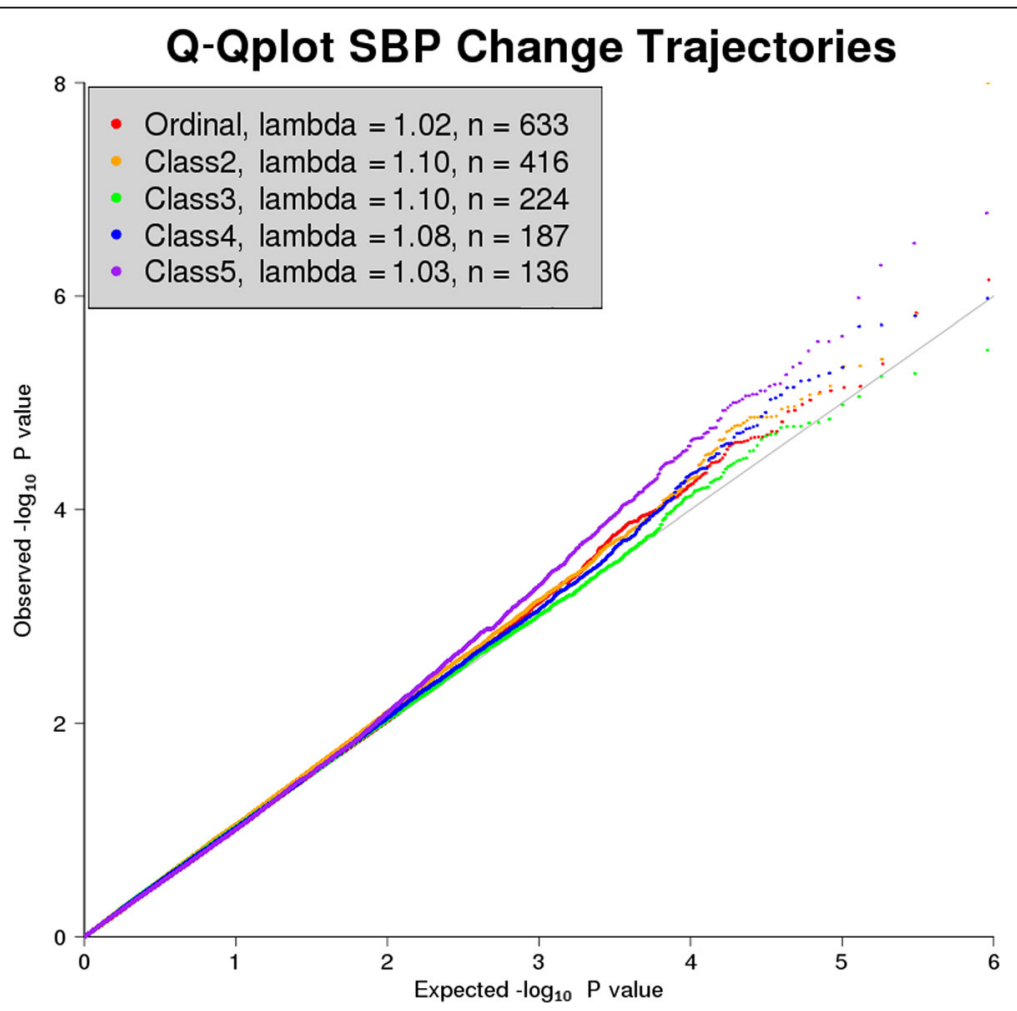

Fig. 3 Q-Q Plot. Quantile-quantile (Q-Q) plot of SNP associations, color-coded by association test, and including sample size (N) and lambda values 
$96 \mathrm{~kb}$ from rs381815, a known SBP-associated SNP identified in Europeans, although in low linkage disequilibrium $\left(r^{2}=0.04\right.$, CEU [Utah residents with ancestry from northern and western Europe] HapMap r22) [15].

\section{Discussion}

We used the SEM model to identify particular time points where certain covariates were more or less informative and direct and indirect effects, which may modify genetic influence on longitudinal SBP in Mexican Americans. While age, sex, and smoking status are known to influence cross-sectional SBP in non-genetic analyses, we found that only age and sex had significant effects on SBP change while accounting for genetic effects of known BP loci. We identified 5 unique trajectories using longitudinal data to create a new outcome variable for genetic association testing. These identified trajectories are significantly heritable, and we identified a total of 8 promising loci that influence one's trajectory in SBP change across age.

One limitation of using the SEM methods to inform covariate selection is that we used previously identified GWA SNPs associated with SBP, DBP, and pulse pressure from cross-sectional data and identified in European, Asian and African descent populations, which may not generalize to Mexican Americans. These loci may not fully account for possible genetic effects in our longitudinal analysis of Mexican Americans and miss possible modifiers to longitudinal genetic effects. The assumption of scale in the ordinal analysis is also a limitation, as health risk may not be equal between the ranked groups. Lastly, another limitation is that GWA study analyses were only performed on odd-numbered chromosomes. We expect that additional loci associated with SBP change will be identified in other regions of the genome in future studies.

\section{Conclusions}

The majority of investigations into the genetic underpinnings of SBP do not take advantage of the wealth of longitudinal data available in many large cohort studies. To address this important research gap, we have capitalized on longitudinal assessments of SBP and extant genetic data available through GAW19 to evaluate a novel and unique statistical data analysis. This study assesses how genetic variants, environment, and behavior effect progression of SBP and will provide new data to examine the pathogenesis of hypertension. The innovative methods considered herein have been used to identify several promising variants associated with SBP change trajectories and can be easily implemented in GWA for a wide range of longitudinally assessed traits.

\section{Acknowledgements}

T2D-GENES is supported by National Institutes of Health (NIH) grants: U01 DK085524, U01 DK085501, U01 DK085526, U01 DK085584, and U01

DK085545. The San Antonio Family Heart Study (SAFHS) is supported by P01
HL045222; the San Antonio Family Diabetes Study (SAFDS) is supported by R01 DK047482; the San Antonio Family Gallbladder Study (SAFGS) is supported by R01 DK053889. This work is in part funded through NIH grant 2 T32 HL007055-36, the American Heart Association Postdoctoral Fellowship, NIMH-MH059490, and U01 HL084756.

\section{Declarations}

This article has been published as part of BMC Proceedings Volume 10 Supplement 7, 2016: Genetic Analysis Workshop 19: Sequence, Blood Pressure and Expression Data. Summary articles. The full contents of the supplement are available online at http://bmcproc.biomedcentral.com/ articles/supplements/volume-10-supplement-7. Publication of the proceedings of Genetic Analysis Workshop 19 was supported by National Institutes of Health grant R01 GM031575.

\section{Authors' contributions}

AEJ conceived of the study, participated in its design and coordination, conducted LCGM and GWA and helped in drafting the manuscript; AGH participated in the study design and coordination, conducted SEM analysis, and helped in drafting the manuscript; GC participated in the study design and coordination, conducted heritability analyses, and helped in GWA analysis and drafting the manuscript; LFR assisted in GWA analysis and drafting the manuscript; MG assisted in statistical analyses and drafting the manuscript; VSV, GD, NF, JO, CLA, and KLY assisted in drafting the manuscript; and KEN participated in its design and coordination, and in drafting the manuscript. AEJ, AGH, and GC contributed equally to this work. All authors read and approved the final manuscript.

\section{Competing interests}

The authors declare that they have no competing interests.

\section{Author details}

'Department of Epidemiology, University of North Carolina, Chapel Hill, NC 27514, USA. ${ }^{2}$ Department of Biostatistics, University of North Carolina, Chapel Hill, NC 27514, USA. ${ }^{3}$ Department of Nutrition, and UNC Nutrition Research Institute, University of North Carolina, Kannapolis, NC 28081, USA.

${ }^{4}$ Department of Statistics, George Mason University, Fairfax, VA 22030, USA.

${ }^{5}$ School of Medicine, University of Maryland, Baltimore, MD 21201, USA.

Published: 18 October 2016

\section{References}

1. Chalmers J, MacMahon S, Mancia G, Whitworth J, Beilin L, Hansson L, Neal B, Rodgers A, Ni Mhurchu C, Clark T. 1999 World Health OrganizationInternational Society of Hypertension Guidelines for the management of hypertension. Guidelines sub-committee of the World Health Organization. Clin Exp Hypertens. 1999;21(5-6):1009-60.

2. Levy D, DeStefano AL, Larson MG, O'Donnell CJ, Lifton RP, Gavras H, Cupples LA, Myers RH. Evidence for a gene influencing blood pressure on chromosome 17. Genome scan linkage results for longitudinal blood pressure phenotypes in subjects from the framingham heart study. Hypertension. 2000;36(4):477-83.

3. Das K, Li J, Fu G, Wang Z, Wu R. Genome-wide association studies for bivariate sparse longitudinal data. Hum Hered. 2011;72(2):110-20.

4. Hossain A, Beyene J. Analysis of baseline, average, and longitudinally measured blood pressure data using linear mixed models. BMC Proc. 2014;8 Suppl 1:S80.

5. Cho SC, Yoo HJ, Park M, Cho IH, Kim BN, Kim JW, Shin MS, Park TW, Son JW, Chung US, et al. Genome-wide association scan of korean autism spectrum disorders with language delay: a preliminary study. Psychiatry Investig. 2011;8(1):61-6.

6. Connolly JJ, Glessner JT, Hakonarson H. A genome-wide association study of autism incorporating autism diagnostic interview-revised, autism diagnostic observation schedule, and social responsiveness scale. Child Dev. 2013;84(1):17-33.

7. Arnedo J, Svrakic DM, Del Val C, Romero-Zaliz R, Hernández-Cuervo H, Molecular Genetics of Schizophrenia Consortium, Fanous AH, Pato MT, Pato $\mathrm{CN}$, de Erausquin GA, et al. Uncovering the hidden risk architecture of the schizophrenias: confirmation in three independent genome-wide association studies. Am J Psychiatry. 2015;172(2):139-53. 
8. Hoadley KA, Yau C, Wolf DM, Cherniack AD, Tamborero D, Ng S, Leiserson MD, Niu B, McLellan MD, Uzunangelov V, et al. Multiplatform analysis of 12 cancer types reveals molecular classification within and across tissues of origin. Cell. 2014;158(4):929-44.

9. Londono D, Chen KM, Musolf A, Wang R, Shen T, Brandon J, Herring JA, Wise CA, Zou H, Jin M, Yu L, et al. A novel method for analyzing genetic association with longitudinal phenotypes. Stat Appl Genet Mol Biol. 2013;12(2):241-61.

10. Jones B, Nagin D, Roeder K. A SAS procedure based on mixture models for estimating developmental trajectories. Sociol Methods Res. 2001;29:374-93.

11. Nagin DS. Group-based trajectory modeling: an overview. Ann Nutr Metab. 2014;65(2-3):205-10.

12. Almasy L, Dyer TD, Peralta JM, Jun G, Wood AR, Fuchsberger C, Almeida MA, Kent Jr JW, Fowler S, Blackwell TW, et al. Data for Genetic Analysis Workshop 18: human whole genome sequence, blood pressure, and simulated phenotypes in extended pedigrees. BMC Proc. 2014;8 Suppl 1:S2.

13. Tobin MD, Sheehan NA, Scurrah KI, Burton PR. Adjusting for treatment effects in studies of quantitative traits: antihypertensive therapy and systolic blood pressure. Stat Med. 2005;24(19):2911-35.

14. Kato N, Takeuchi F, Tabara Y, Kelly TN, Go MJ, Sim X, Tay WT, Chen CH, Zhang $Y$, Yamamoto $K$, et al. Meta-analysis of genome-wide association studies identifies common variants associated with blood pressure variation in east Asians. Nat Genet. 2011;43(6):531-8.

15. Levy D, Ehret GB, Rice K, Verwoert GC, Launer $\sqcup$, Dehghan A, Glazer NL, Morrison AC, Johnson AD, Aspelund T, et al. Genome-wide association study of blood pressure and hypertension. Nat Genet. 2009;41(6):677-87.

16. Newton-Cheh C, Johnson T, Gateva V, Tobin MD, Bochud M, Coin L, Najjar SS, Zhao JH, Heath SC, Eyheramendy S, et al. Genome-wide association study identifies eight loci associated with blood pressure. Nat Genet. 2009; 41(6):666-76.

17. He J, Kelly TN, Zhao Q, Li H, Huang J, Wang L, Jaquish CE, Sung YJ, Shimmin LC, Lu F, et al. Genome-wide association study identifies 8 novel loci associated with blood pressure responses to interventions in Han Chinese. Circ Cardiovasc Genet. 2013;6(6):598-607.

18. Franceschini N, Kelly TN, Zhao Q, Li H, Huang J, Wang L, Jaquish CE, Sung YJ, Shimmin LC, Lu F, et al. Genome-wide association analysis of bloodpressure traits in African-ancestry individuals reveals common associated genes in African and non-African populations. Am J Hum Genet. 2013;93(3):545-54

19. Bhatnagar P, Barron-Casella E, Bean CJ, Milton JN, Baldwin CT, Steinberg MH, Debaun M, Casella JF, Arking DE. Genome-wide meta-analysis of systolic blood pressure in children with sickle cell disease. PLoS One. 2013;8(9):e74193.

20. Hu LT, Bentler PM. Cutoff criteria for fit indexes in covariance structure analysis: conventional criteria versus new alternatives. Struct Equ Modeling. 1999;6(1):1-55

21. Bentler PM. Comparative fit indexes in structural models. Psychol Bull. 1990;107(2):238-46.

22. Tucker L, Lewis C. A reliability coefficient for maximum likelihood factor analysis. Psychometrika. 1973;38(1):1-10.

23. Nagin DS. Analyzing developmental trajectories: a semiparametric, groupbased approach. Psychol Methods. 1999;4(2):139-57.

24. Nagin D. Group-based modeling of development. Cambridge: Harvard University Press; 2005 .

25. Andruff H, Carraro N, Thompson A, Gaudreau P. Latent class growth modelling: a tutorial. Tutor Quant Methods Psychol. 2009;5(1):11-24.

26. Price AL, Patterson NJ, Plenge RM, Weinblatt ME, Shadick NA, Reich D. Principal components analysis corrects for stratification in genome-wide association studies. Nat Genet. 2006;38(8):904-9.

27. Quillen EE, Voruganti VS, Chittoor G, Rubicz R, Peralta JM, Almeida MA, Kent Jr JW, Diego VP, Dyer TD, Comuzzie AG, et al. Evaluation of estimated genetic values and their application to genome-wide investigation of systolic blood pressure. BMC Proc. 2014;8 Suppl 1:S66.

28. Almasy L, Blangero J. Multipoint quantitative-trait linkage analysis in general pedigrees. Am J Hum Genet. 1998;62(5):1198-211.

29. O'Connell JR. MMAP user guide. Baltimore: University of Maryland; 2014.

30. Voruganti VS, Kent Jr JW, Debnath S, Cole SA, Haack K, Göring HH, Carless MA, Curran JE, Johnson MP, Almasy L, et al. Genome-wide association analysis confirms and extends the association of SLC2A9 with serum uric acid levels to Mexican Americans. Front Genet. 2013;4:279.

\section{Submit your next manuscript to BioMed Central and we will help you at every step:}

- We accept pre-submission inquiries

- Our selector tool helps you to find the most relevant journal

- We provide round the clock customer support

- Convenient online submission

- Thorough peer review

- Inclusion in PubMed and all major indexing services

- Maximum visibility for your research

Submit your manuscript at www.biomedcentral.com/submit

) Biomed Central 